\title{
Indomethacin retinopathy: case report and review
}

\author{
C M GRAHAM AND R K BLACH \\ From Moorfields Eye Hospital, City Road, London EC1V 2PD
}

SUMMARY The case of a 33-year-old man with probable indomethacin retinopathy is presented. The relevant literature is reviewed and the differences between our case and those previously reported are noted. Our patient appears to have suffered severe and irreversible ocular damage due to the very high dose of indomethacin ingested over a prolonged period.

A 33-year-old man was seen in February 1986. He had noticed a gradual reduction in visual acuity in both eyes for 18 months becoming more noticeable over the past nine months. In addition he gave an 18month history of difficulty with night vision and increasing photophobia. He had worn glasses since the age of 18 for correction of low myopia $(-1 \cdot 0 \mathrm{D} \mathrm{R}$ and $L$ ) and reported that in 1978 his optician was unable to improve his right visual acuity to $6 / 6$; an abnormal appearance was noted at the back of that eye. His general health was good, but for eight years up to the age of 25 he took indomethacin $200 \mathrm{mg}$ per day for arthritis of his neck. Subsequently this was taken on an occasional basis for another year, stopping completely in 1979 . No other regular medication was taken in conjunction with this and he had never taken chloroquine. There was no family history of eye trouble and no consanguinity.

On examination visual acuity in each eye was $6 / 24$ with glasses but could be improved to $6 / 18$ with the aid of a pinhole. Colour vision was profoundly affected, and he could read only the first of the 17 numbers on the Ishihara plates. The anterior segments were normal, and there were no corneal deposits. There was no relative afferent pupillary defect. The intraocular pressures were normal. There was retinal thinning with pigment mottling, especially in the macular region of both eyes.

Fluorescein angiography confirmed these findings in a rather dramatic fashion and showed a bullseye appearance of the macula (Fig. 1). Electrodiagnostic tests showed a reduced electro-oculogram (EOG) (right $125 \%$ and left $140 \%$ ), and the electroretino-

Correspondence to C M Graham FRCS. gram (ERG) was virtually absent with no oscillatory potential and minimal flicker (Fig. 2).

Visual field assessment with a Goldmann perimeter revealed bilateral central scotomata with constriction to I4 targets (Fig. 3), and a diffuse abnormality in colour vision was demonstrated on the 100-hue test (Fig. 4). A diagnosis of indomethacin retinopathy was made.

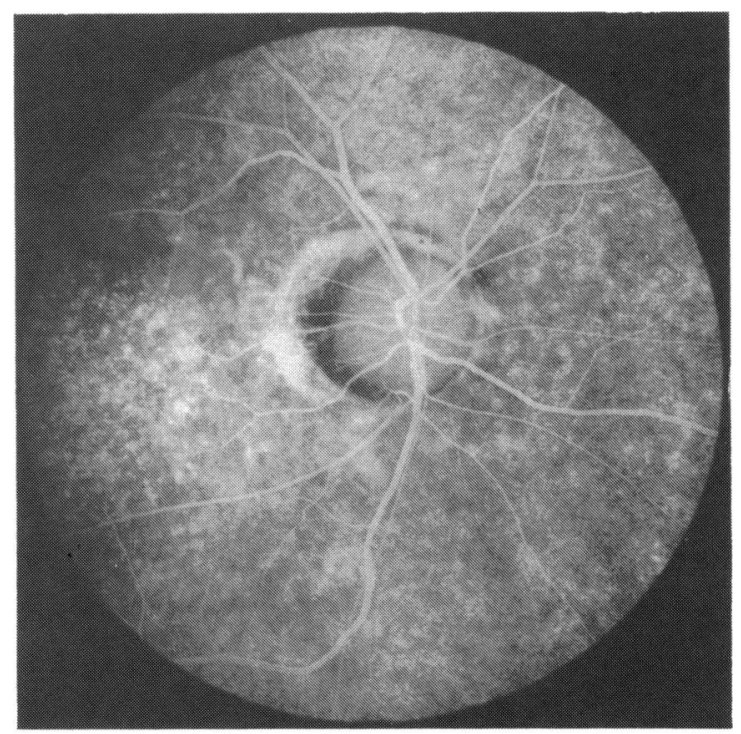

Fig. 1A

Fig. 1 Fluorescein angiogram of (A, B) right optic disc and macula $(\mathrm{C})$ left optic disc and macula. There is pigmentary mottling with a bullseye maculopathy. 


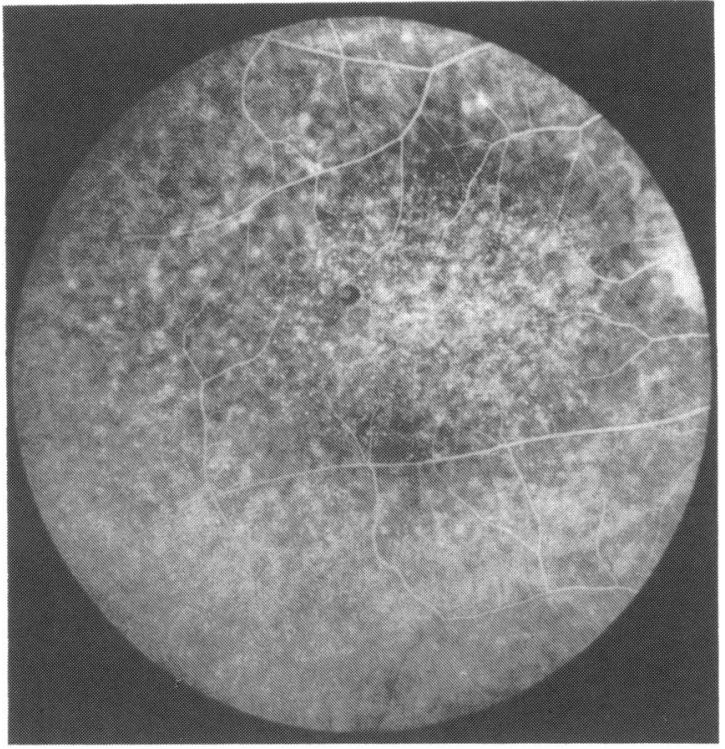

Fig. 1B

\section{Discussion}

\section{PREVIOUS REPORTS}

Indomethacin, which was first introduced in 1963, is a non-steroidal anti-inflammatory agent which has analgesic and antipyretic properties. It has been used for a wide variety of inflammatory and degenerative joint conditions, acute musculoskeletal disorders, and acute gout.

Despite its widespread, frequent, and often pro-

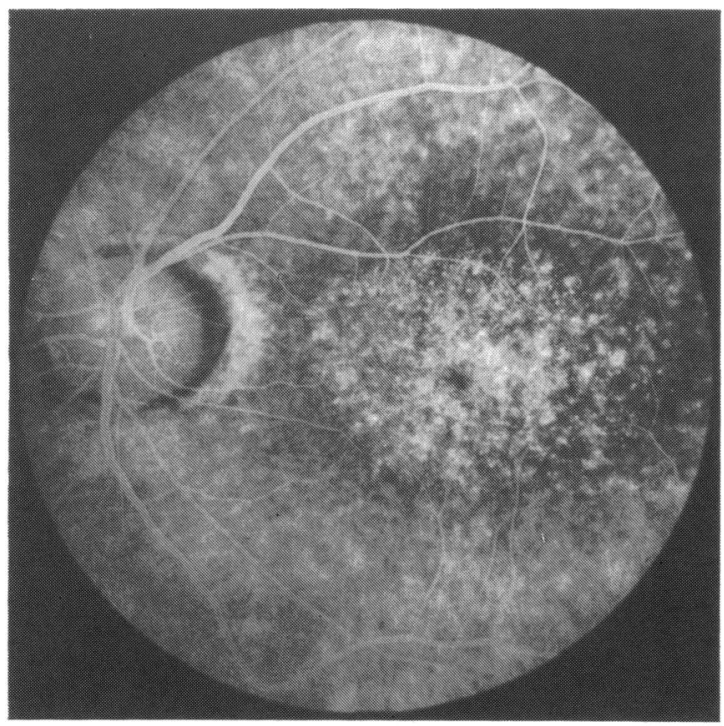

Fig. 1C
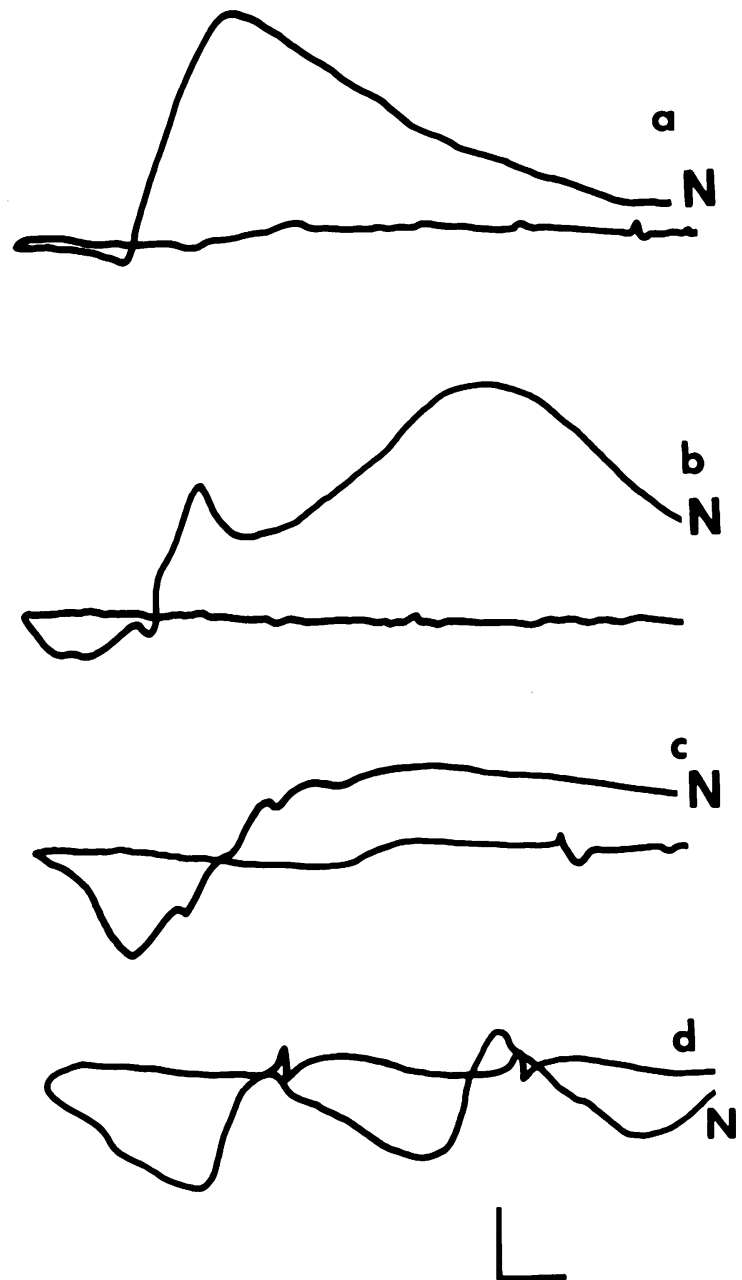

Fig. 2 Electroretinogram of subject compared with normal (N): (a) blue light stimulus, (b) red light stimulus, (c) white light stimulus, (d) response to a $30 \mathrm{~Hz}$ flicker. There is an almost absent ERG and minimal flicker. Calibration mark: Gain $100 \mu V$ per division for trace (a), (c), and (d) and $50 \mu V$ per division for trace (b). Sweep speed scale: $20 \mathrm{~ms} \mathrm{per}$ division for trace (a) and (b) and $10 \mathrm{~ms}$ per division for trace (c) and (d).

longed use, ophthalmic side effects seem to be rare. Burns' first reported corneal deposits and changes in the retinal pigment epithelium in a 54-year-old man in 1966 and followed this with a series of patients two years later. ${ }^{2} \mathrm{~A}$ further case of retinopathy has been described by Henkes et $a l .,{ }^{3}$ and the ocular side effects in small series of patients have been reported by Carr and Siegel ${ }^{4}$ and Palimeris et al. ${ }^{5}$ Acquired colour vision defects $^{67}$ and electrodiagnostic changes ${ }^{89}$ have been reported.

A reduction in visual acuity to $20 / 70$ was reported 


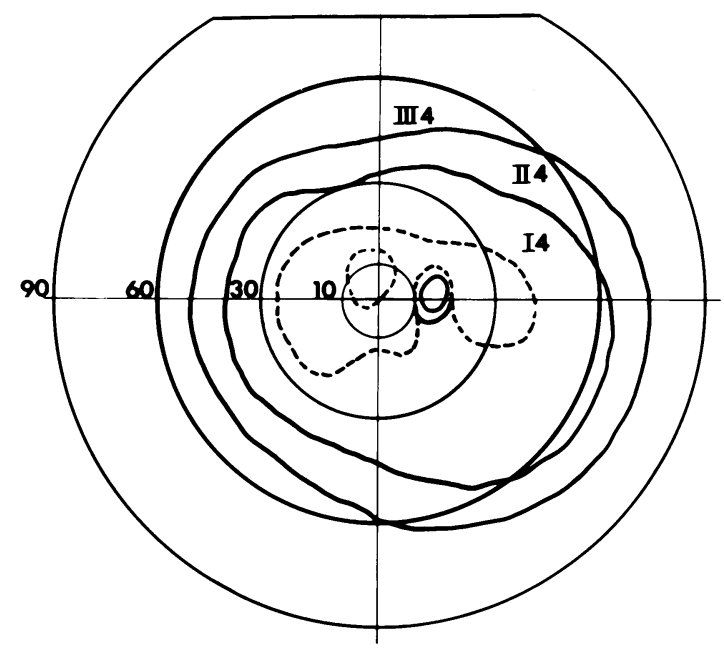

Fig. 3A

Fig. 3 Goldmann visual fields: (A) right eye, (B) left eye. There is a bilateral central scotoma and field constriction to 14 targets.

by Burns' in her original case report, but this returned to 20/20 following withdrawal of treatment. The patient reported by Henkes et al. ${ }^{3}$ had a worsening of visual acuity on treatment with indomethacin, but there was only a partial recovery off treatment. In the series of 18 patients treated with indomethacin described by Palimeris et al. ${ }^{5}$ visual acuity was reduced in the three patients noted to have macular changes but normal in the others.

No significant alteration in colour vision was reported by Grützner ${ }^{6}$ in a patient who had also

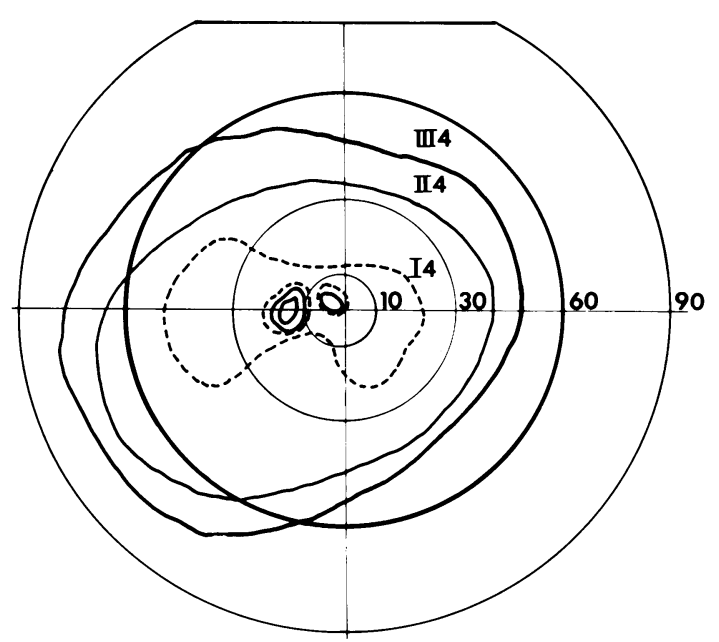

Fig. 3B

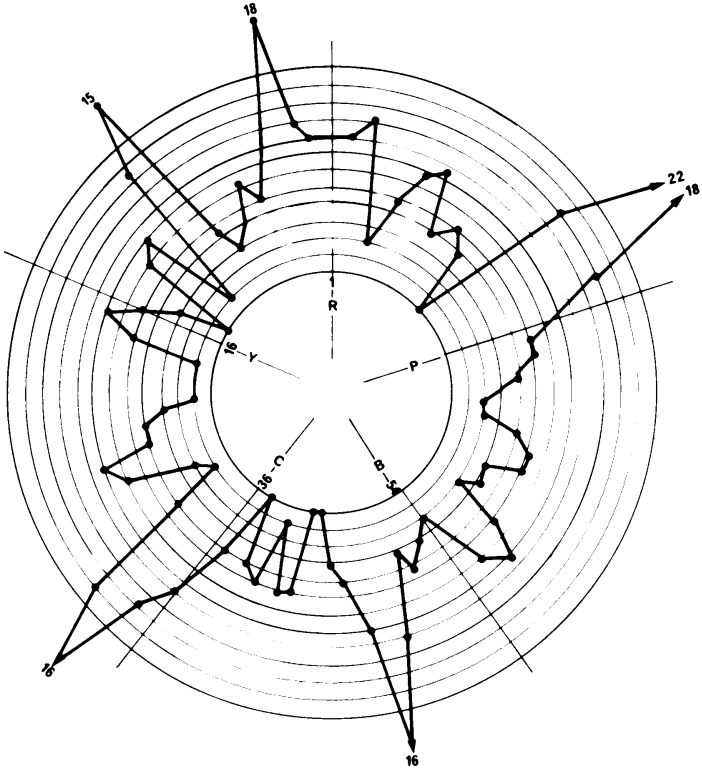

Fig. 4A

Fig. 4 Farnsworth-Munsell 100-hue test demonstrating a diffuse colour defect, (A) right eye, (B) left eye.

received chloroquine, nor in a series of 18 patients receiving indomethacin only, when compared with a control group. ${ }^{4}$ Abnormalities have been noted by Burns in one patient, and in that particular case the abormality was unilateral and associated with a central serous detachment. The only reports of

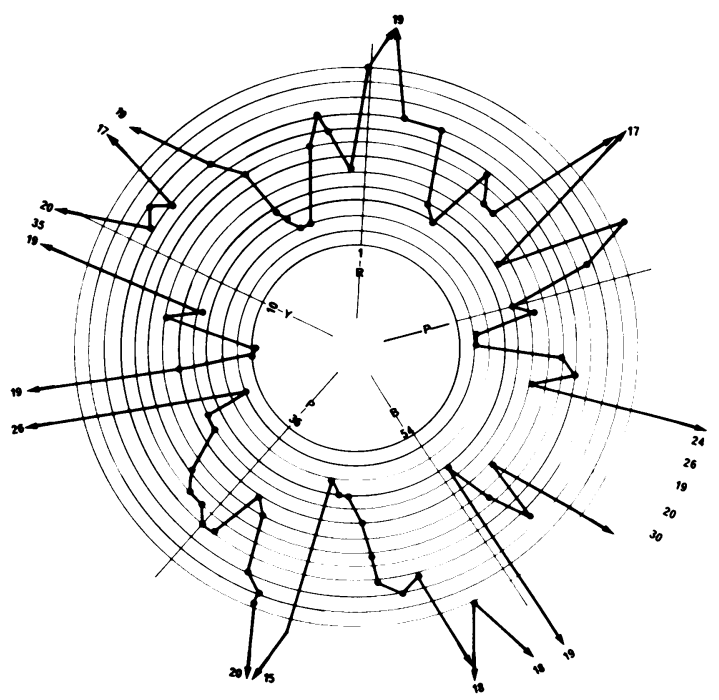

Fig. 4B 
definite colour vision changes have come from Palimeris et al., ${ }^{5}$ who noted two patients developing acquired defects of the blue-yellow axis after 15 and 17 months of treatment with indomethacin. Both returned to normal after stopping treatment. In a second report from the same department ${ }^{7}$ two out of 11 patients were noted to have an acquired blueyellow defect and one had a diffuse defect of no prominent axis. Again these had returned to almost normal after withdrawal of treatment.

Conflicting reports exist for field changes. Burns described three patients with field constriction and paracentral scotomata with significant improvement following withdrawal of treatment, and similarly, Henkes et al. ${ }^{3}$ described constriction of field and enlargement of the blind spot during treatment followed by partial recovery. In the two other series no visual field changes nor scotomata were noted," except for one patient with a mild constriction of the visual fields attributed to moderate myopia ( $-6 \cdot 0$ Dsph R and L). ${ }^{4}$

Macular disturbances were reported in 10 out of 34 cases by Burns. ${ }^{2}$ These retinal changes were nonspecific and included two cases of macular pucker and a case of central serous retinopathy. The majority, however, were described as having paramacular depigmentation varying in severity from a mottled depigmentation to areas of pigment atrophy. Visual acuity was reduced in all these patients.

It was emphasised that some of these pigmentary changes were very subtle and sometimes best revealed by fluorescein angiography. Carr and Siegel ${ }^{4}$ demonstrated no significant ophthalmoscopic changes and no specific features on fluorescein. Palimeris et al. ${ }^{5}$ noted macular abnormalities in three out of 18 patients. Two of these had perifoveal accumulation of pigment, loss of foveal reflex, and areas of depigmentation round the macula. One case had diffuse perifoveal pigmentation. Although there was some improvement in the ophthalmoscopic picture one year after withdrawal of treatment, the depigmented areas around the maculae remained.

Electrodiagnostic changes have been noted in indomethacin retinopathy. Burns ${ }^{2}$ noted a decrease in retinal sensitivity in all cases as shown by a decreased ERG, and Henkes et al. ${ }^{39}$ noted a deterioration of the scotopic ERG and the EOG ratio which improved slowly after withdrawal of treatment to the levels found at the first examination while the patients were taking indomethacin. It was pointed out that this could be interpreted as indicative of a good prognosis, but the visual acuity did not reflect this, and the deep pigmentary changes did not alter significantly. In their study of a selected group of patients who had taken indomethacin for between 12 and 18 months Palimeris et al. ${ }^{58}$ found that indo- methacin reduced the amplitude of the ERG in $18 \%$ cases, with the scotopic element being more affected than the photopic. The $a$ and $b$ waves of the ERG improved following withdrawal of the drug. In the same group of patients two were found to have an abnormal EOG, with the L/D ratio reduced to $160 \%$ in both cases.

One case returned to normal after indomethacin was stopped but the other remained unchanged. They could detect no correlation between ERG and EOG nor could they establish a relationship between dose and signs of toxicity, but they suggested that a total of at least $45 \mathrm{~g}$ was needed after one year of treatment. Carr and Siegel ${ }^{4}$ could detect no significant difference between their patients and control group of patients, and attributed any abnormalities of the ERG to retinal and choroidal arteriosclerotic changes.

\section{PRESENT CASE}

The most striking differences between our patient and the few described in the literature lie in the deterioration in vision several years after withdrawal of indomethacin and the gross changes in the ERG and EOG, which might have been expected to improve. The fluorescein appearance was also particularly striking.

Our patient either has a more severe retinopathy than has previously been reported or a second disease, with indomethacin ingestion being either coincidental or only partly contributory. Chloroquine had never been taken, and we also carefully considered the possibility of cone-rod dystrophy but dismissed it. The macular pigmentary changes were rather more diffuse than usually seen in this condition and more compatible with a toxic retinopathy. In addition there were no significant changes in the peripheral fundus. Although it is impossible to be absolutely certain, we believe that our patient does have an indomethacin retinopathy, with many features qualitatively similar to those described by other authors.

Abnormalities were detected in our patient while he was taking indomethacin, but there was continued deterioration following withdrawal of the treatment. This fact does not, however, exclude indomethacin as a cause of toxicity, since other drugs, notably chloroquine, are known to cause progressive changes long after withdrawal. Indomethacin differs from chloroquine, however, in that it is excreted much more rapidly from the body.

The changes which have been reported elsewhere to be reversible on withdrawal of indomethacin may not be reversible if higher total doses of the drug are ingested. Carr and Siegel, ${ }^{4}$ who could demonstrate no retinopathy, looked at patients with a mean dose 
of $32.8 \mathrm{~g}$, whereas Burns ${ }^{211}$ in her series looked at patients taking a mean dose of $56.5 \mathrm{~g}$. The patient described by Henkes et al. ${ }^{3}$ had taken a total dose of $181 \mathrm{~g}$ indomethacin in the form of suppositories. The serum concentration, they pointed out, is approximately $50 \%$ lower than following the oral ingestion of an equal dose. Our patient, in contrast, had taken over $550 \mathrm{~g}$ orally during the eight years of regular use and an additional unknown quantity subsequently when he took indomethacin on a less regular basis.

There is a great deal of uncertainty regarding the degree to which indomethacin is retinotoxic. We consider there is sufficient evidence to incriminate it, and it is interesting that both the British National Formulary and MIMS (Monthly Index of Medical Specialities) state that patients undergoing prolonged therapy should undergo periodic ophthalmic examinations. In clinical practice this is the exception rather than the rule, and further work is needed to evaluate this advice concerning a drug which is widely used.

We are grateful to the staff of the Electrodiagnostic Clinic at Moorfields Eye Hospital for their assistance in recording the electroretinograms and electro-oculograms.

\section{References}

1 Burns CA. Ocular effects of indomethacin. Slit lamp and electroretinographic (ERG) study. Invest Ophthalmol Vis Sci 1966; 5: 325 .

2 Burns CA. Indomethacin, reduced retinal sensitivity and corneal deposits. Am J Ophthalmol 1968; 66: 825-35.

3 Henkes HE, van Lith GHM, Canta LR. Indomethacin retinopathy. Am J Ophthalmol 1972; 73: 846-56.

4 Carr RE, Siegel IM. Retinal function in patients treated with indomethacin. Am J Ophthalmol 1973; 75: 302-6.

5 Palimeris G, Koliopoulos J, Velissaropoulos P. Ocular side effects of indomethacin. Ophthalmologica 1972; 164: 339-53.

6 Grützner P. Acquired colour vision defects secondary to retinal drug toxicity. Ophthalmologica 1969; 158: (suppl): 592-604.

7 Koliopoulos J, Palimeris G. On acquired colour vision disturbances during treatment with ethambutol and indomethacin. Mod Probl Ophthalmol 1972; 11: 178-84.

8 Palimeris G, Koliopoulos J, Velissaropoulos P. Electrophysiological findings in patients treated with indomethacin. Adv Exp Med Biol 1972; 24: 323-9.

9 Henkes HE, Deutman AF. Electrodiagnostic procedures in drug induced ocular diseases. Doc Ophthalmol 1973; 34: 209-19.

10 Burns CA. Indomethacin induced ocular toxicity. Am J Ophthalmol 1973; 76: 312-3.

Accepted for publication 23 April 1987. 